\title{
Mitochondrial abnormalities: a hub in metabolic syndrome-related cardiac dysfunction caused by oxidative stress
}

\author{
Aoxue $\mathrm{Li}^{1,2} \cdot$. Ningning Zheng ${ }^{3} \cdot$ Xudong Ding $^{4}$
}

Accepted: 13 April 2021 / Published online: 5 May 2021

(c) The Author(s) 2021

\begin{abstract}
Metabolic syndrome (MetS) refers to a group of cardiovascular risk elements comprising insulin resistance, obesity, dyslipidemia, increased glucose intolerance, and increased blood pressure. Individually, all the MetS components can lead to cardiac dysfunction, while their combination generates additional risks of morbidity and mortality. Growing evidence suggests that oxidative stress, a dominant event in cellular damage and impairment, plays an indispensable role in cardiac dysfunction in MetS. Oxidative stress can not only disrupt mitochondrial activity through inducing oxidative damage to mitochondrial DNA, RNA, lipids, and proteins but can also impair cardiomyocyte contractile function via mitochondria-related oxidative modifications of proteins central to excitation-contraction coupling. Furthermore, excessive reactive oxygen species (ROS) generation can lead to the activation of several mitochondria apoptotic signaling pathways, release of cytochrome c, and eventual induction of myocardial apoptosis. This review will focus on such processes of mitochondrial abnormalities in oxidative stress induced cardiac dysfunction in MetS.
\end{abstract}

Keywords Oxidative stress $\cdot$ Mitochondria $\cdot$ Reactive oxygen species $\cdot$ Metabolic syndrome $\cdot$ Cardiac dysfunction

\section{Introduction}

Metabolic syndrome (MetS) refers to a group of cardiovascular risk factors comprising insulin resistance, obesity, dyslipidemia, increased glucose intolerance, and increased blood pressure [1]. MetS components can act independently as risk factors for cardiovascular disease (CVD), while their combination may significantly increase the morbidity and severity of cardiovascular conditions. When

Aoxue Li and Ningning Zheng are co-authors and contribute equally to the article.

Xudong Ding

dingxd@sj-hospital.org

1 Department of Pharmaceutical Biotechnology, China Medical University-The Queen's University of Belfast Joint College, China Medical University, Liaoning, China

2 Covance Pharmaceutical Research and Development (Beijing) Co, Ltd, Beijing, China

3 Department of Pathophysiology, College of Basic Medical Science, China Medical University, Liaoning, China

4 Department of Anesthesiology, Shengjing Hospital, China Medical University, Liaoning, China cardiac dysfunction occurs in MetS patients with coronary artery disease or other etiologies, it suggests that they may suffer specific cardiomyopathies, including obesity-related and diabetic cardiomyopathy and insulin resistance-related cardiac dysfunction [2]. Controlling such cardiovascularrelated injuries is an important strategy to alleviate MetS clinical mortality. A state of elevated oxidative stress appears to be a central mechanism underlying the pathophysiology of MetS and related CVD [2]. Excessive reactive oxygen species(ROS) induce irreversible structural and functional impairment in cardiac myocytes, involving multiple pathological cardiovascular conditions, and also been widely known as a key contributor for cardiac hypertrophy and remodeling through stimulating signal transduction which controls cardiomyocytes apoptosis and necrosis. The key role of mitochondria in the production of ROS and regulation of cardiomyocyte survival and heart pump function determines that mitochondria dysfunction is a great stimulus triggering almost all pathological aspects of oxidative stress induced heart dysfunction. Medium and later stage of cardiac hypertrophy with decreased amounts of mitochondria usually accompanies with contractile dysfunction [3]. Mitochondrial structure damage and function decline form a catastrophic cycle 
with ROS and continue to advance the progression of the disease. In this review, we are just on the perspective of this hub, mitochondria, to summarize recent insights into the mechanisms of oxidative stress-related cardiac dysfunction from mitochondrial damage, cardiomyocyte contractile dysfunction, and apoptosis (Fig. 1).

\section{Oxidative stress and mitochondrial damage}

Heart is a high-energy demand organ with extremely dependence on the normal structure and function of mitochondria [4]. Mitochondria represent a primary source of ROS and also suffer ROS-mediated damage, suggesting that a pathophysiological relationship exists between oxidative stress and mitochondrial dysfunction [5, 6]. Maintaining ROS generation and removal homeostasis is a pivotal prerequisite for ensuring mitochondria metabolic adaption. In turn, the pathological process of excessive ROS is characterized by mitochondrial abnormality, including enhanced peroxidation of mitochondrial lipids, decreased mitochondrial DNA (mtDNA) and mitochondrial RNA (mtRNA) copy numbers, and the reduced oxidizing ability [7].

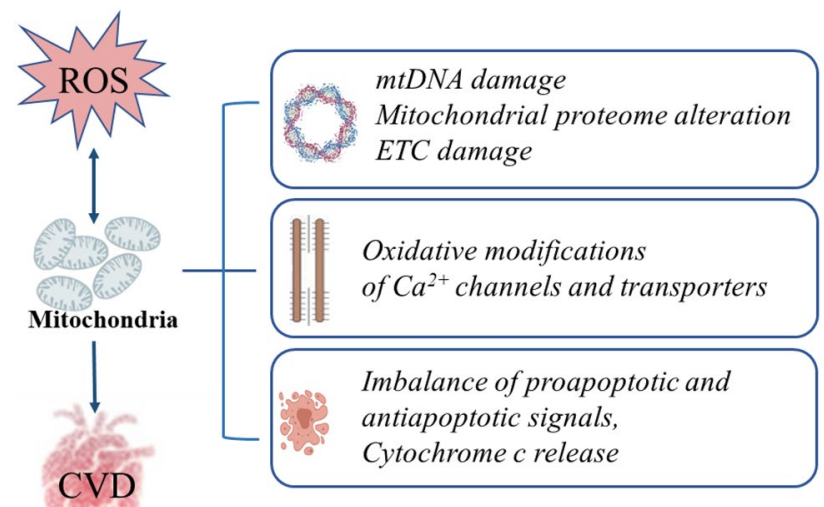

Fig. 1 Mitochondria act as a key regulator in metabolic heart disease caused by oxidative stress. Three possible mechanisms of mitochondria involving in this process are summarized: ROS mediate oxidative damage to mitochondrial DNA, RNA, and proteins, resulting in ETC function impairment. The mitochondria-related oxidative modifications of $\mathrm{Ca}^{2+}$ channels and transporters can directly cause cardiomyocyte systolic and diastolic dysfunction. Excessive ROS induce mitochondria-dependent cardiomyocyte apoptosis by activating proapoptotic signaling pathways, suppressing antiapoptotic signaling pathways, and mediating cytochrome $\mathrm{c}$ release. ROS reactive oxygen species, CVD cardiovascular disease, mtDNA mitochondrial DNA, ETC electron transport chain

\section{Mitochondrial DNA damage}

Oxidative stress has destroyed mitochondrial macromolecules both at and near the sites of their formation, specifically induced oxidative damage to mtDNA, mtRNA, lipids, and proteins, which eventually contributes to mitochondrial dysfunction [7]. Mitochondria have their own DNA which encodes 13 subunits of mammalian respiratory complexes, as well as 22 tRNAs and 2 rRNAs [8]. mtDNA exhibits a higher sensitivity to oxidative damage than nuclear DNA, possibly because mtDNA is closer to ROS-generating respiratory chains, while also lacking the protection of histone-like proteins and the ability to repair DNA damage [9]. Excessive ROS production in mitochondria can progressively destroy mtDNA, leading to a concomitant reduction in mitochondrial RNA and protein levels, and eventually to the loss of mitochondrial function [10]. This is also supported by in vitro studies on cultured vascular endothelial and smooth muscle cells, which demonstrated that excessive ROS generation can lead to mtDNA damage, gene expression alterations, and mitochondrial dysfunction [10]. Regarding mtDNA damage, ROS accumulation has a higher propensity to induce the generation of basic sites and strand breaks than mutagenic base lesions, which may be related to the ability of mitochondria to retain their genetic integrity. If mitochondrial base excision repair pathway components fail to correctly repair mtDNA damage by removing the damaged DNA bases and abasic sites, mtDNA will become unstable and degraded, eventually resulting in mitochondrial dysfunction [11]. Specifically, superoxide anions $\left(\mathrm{O}^{2 \bullet-}\right)$, one of the major ROS, are produced by the mitochondrial electron transport chain (ETC) through the reduction of oxygen [12]. These anions are then released into the mitochondrial matrix and intermembrane space and converted either spontaneously or enzymatically to hydrogen peroxide $\left(\mathrm{H}_{2} \mathrm{O}_{2}\right)$. Finally, $\mathrm{H}_{2} \mathrm{O}_{2}$ diffuses throughout the cell and generates highly reactive hydroxyl radicals with a strong capacity to damage mtDNA [13]. Studies have shown that the main mtDNA modifications resulting from ROS-mediated insult are the generation of 7,8-dihydro-8-oxo-2-deoxyguanosine (8-oxoG) among purines [14] and thymine glycol among pyrimidines [15]. In addition, excessive mitochondrial ROS generation may result in a vicious cycle of declining mitochondrial function, whereby mitochondrial ROS causes mtDNA damage and mutations, which conversely exacerbate the injury to respiratory chain function and induce subsequent ROS accumulation. The decreased mtDNA copy number and subsequent mtDNA defects due to excessive ROS production lead to a decline in mitochondrial transcript and protein levels, as well as the impairment of ETC function 
[7]. Consequently, mitochondrial dysfunction contributes to increasing mitochondrial oxidative injury, impairing mitochondrial respiration, and disturbing mitochondrial substrate utilization, which may eventually lead to cardiac dysfunction.

\section{Mitochondrial proteome alteration}

Alteration of the mitochondrial proteome and associated mitochondrial dysfunction is implicated in heart disease. It has been demonstrated that an underlying connection exists between oxidative stress and changes in the cardiac mitochondrial proteome [16], although the relationship has yet to be extensively investigated. In a rat streptozotocin model of chronic type I diabetes, myocardial oxidative stress and concomitant proteome variations in diabetic hearts were shown to proceed synchronously, and $50 \%$ of proteins exhibiting altered expression were localized to mitochondria [16]. Additionally, a different study conducted by Dai et al. also reported the central roles of mitochondrial oxidative stress in proteome remodeling under heart failure induced by pressure overload, with dramatically altered 96 proteins like reduced fatty acid metabolic proteins and elevated glycolytic enzymes in the cardiac ventricular tissues of wild type mice with transverse aortic constriction (TAC)-induced heart failure compared with wild-type controls. Among them, 28\% changed proteins are metabolic proteins which are mainly involved in fatty acid metabolism, like Acyl-CoA dehydrogenases; $23 \%$ of changed proteins are ETC proteins; and $30 \%$ of the proteome remodeling is comprised by proteins participated in transmembrane transport, ATP generation, protein folding, and proteolysis, as well as apoptosis [17]. Interestingly, regarding the changes of ETC complexes, the abundance of various subunits was observed to change inconsistently [17]. The inconsistency was also previously reported by Bugger et al. that over $50 \%$ of ETC proteins decreased with some subunits of complexes I, II, and V increased after TAC-induced heart failure [18]. In contrast, Dai et al. found that the increased subunits are more than decreased ones after TAC, and suggested that the increased abundance of these ETC components seems to lead to more dysfunctional damaged proteins [17], which is in opposition to most studies considering the declined mitochondrial respiratory function in heart failure is associated with susceptibility of ETC complexes to oxidative damage. However, mitochondrial catalase-mediated scavenging of mitochondrial ROS was demonstrated that can greatly attenuate the mitochondrial proteome remodeling in response to TAC [17]. Therefore, an increased understanding of the relationship between mitochondrial proteomic changes and oxidative stress may be important for predicting and analyzing the therapeutic response to both established and novel antioxidant therapeutics for the treatment of heart failure. When cardiovascular disease occurs, the transformation of metabolic substrates during the process of mitochondrial energy metabolism, changes in metabolic mode, and related mitochondrial metabolomics modification changes involved in these processes will be further clarified with the in-depth analysis of sequencing technology.

\section{Oxidative stress and mitochondria- dependent heart pump dysfunction}

The normal contraction and relaxation of cardiomyocytes depend on the appropriately adjusted calcium ions level in the cytoplasm, the combined state of calcium and troponin, and the formation of cross bridges. Excessive oxidative stress can directly cause cardiomyocyte systolic and diastolic dysfunction by inducing oxidative posttranslational modifications of proteins involved in excitation-contraction coupling (ECC) in MetS hearts [19]. ECC describes the conversion of electrostimulation, such as an action potential, to a mechanical response in a single cardiomyocyte. In this accurate tuned process of electrostimulation, intracellular sodium ions $\left(\mathrm{Na}^{+}\right)$and calcium ions $\left(\mathrm{Ca}^{2+}\right)$ play central roles [20]. Deficient $\mathrm{Ca}^{2+}$ and $\mathrm{Na}^{+}$handling has been suggested to result in oxidative stress by initiating a vicious cycle that affects ECC and leads to cardiac dysfunction and heart failure [21]. Mitochondrial calcium uptake plays a very important role in maintaining the normal physiological functions of cells, including stimulating ATP production, inhibiting autophagy, rectifying intracytoplasmic calcium signals, and regulating cell death. Mitochondrial calcium uniporter (MCU) are important mediators of mitochondrial calcium integration signals. Studies suggest that the regulation of mitochondrial calcium uptake 1 (MICU1) and mitochondrial calcium uptake 2 (MICU2) on MCU activity involves a gating mechanism: when the cell is in a resting state and the concentration of $\mathrm{Ca}^{2+}$ in the cytoplasm is low, MICU1-MICU2 inhibit $\mathrm{Ca}^{2+}$ from entering the mitochondria through the MCU. When the cell is stimulated by signals and the concentration of $\mathrm{Ca}^{2+}$ in the cytoplasm rises and exceeds a certain threshold (about greater than $1 \mu \mathrm{M})$, MICU1-MICU2 allow $\mathrm{Ca}^{2+}$ to enter the mitochondria through the MCU. This gating mechanism not only effectively prevents mitochondria from ingesting excessive $\mathrm{Ca}^{2+}$ in the resting state of the cell, leading to oxidative damage and even cell death, but also enables mitochondria to respond to and rectify cytoplasmic calcium signals to complete normal cellular physiological processes. Heart disease has been found to be related to mitochondrial calcium disorders. Ming-Feng Tsai's study shows that the formation of MCU complex may contribute to its localization at the mitochondrial inner and outer membrane contact point, thereby promoting mitochondrial uptake of $\mathrm{Ca}^{2+}$ 
from the cytoplasm, which elucidates the molecular mechanism by which mitochondria regulate calcium uptake in response to cytoplasmic calcium signals [22]. Sarcoplasmic/ endoplasmic reticulum calcium ATPase 2a (SERCA2a), which is expressed in cardiac muscle and controls its relaxation through sequestering $\mathrm{Ca}^{2+}$, displays impaired activity due to increased oxidative stress in the MetS heart, which eventually leads to cardiac diastolic dysfunction. The underlying mechanism was shown to be that posttranslational modification of SERCA2a induced by ROS overproduction results in prolonged $\mathrm{Ca}^{2+}$ transients and slower SERCA2amediated $\mathrm{Ca}^{2+}$ reuptake [23]. ROS accumulation gives rise to cardiac $\mathrm{Ca}^{2+}$ overload through redox modulation of ion channel and ion pump activity [24]. Regarding the targets for oxidative modifications, the sulfhydryl groups of cysteine residues have been shown to be critical targets for oxidative modification. The oxidative posttranslational modifications can elicit diverse functional results depending on the type of pump and ion channels, as well as other transporter types. For instance, the redox modification of thiol groups can hyperactivate cardiac ryanodine receptor (RyR) which is a redox-sensitive sarcoplasmic reticulum $\mathrm{Ca}^{2+}$ release channel located in the inner mitochondrial membrane (IMM) [25, 26], leading to calcium leak, but it can also inhibit SERCA activity [27], thereby directly changing calcium kinetics. As a result, the altered calcium kinetics disrupt the mechanisms of force activation, generation, and transmission. In addition, mitochondrial $\mathrm{Ca}^{2+}$ overload is associated with elevated ROS release and decreased ATP generation in heart failure. Specifically, intramitochondrial calcium contributes to maintaining energy metabolism homeostasis and cardiac function via activating Krebs cycle dehydrogenases; however, this process is disrupted in heart failure, and the impaired mitochondrial $\mathrm{Ca}^{2+}$ uptake results in NADPH oxidation, excessive ROS production, and ultimately cardiac dysfunction [21]. On the other hand, pirfenidone has been demonstrated that it cannot only enhance the contractility of cardiomyocytes by promoting myofilament $\mathrm{Ca}^{2+}$ desensitization and increasing $\mathrm{Ca}^{2+}$ uptake but also inhibiting ROS generation so as to attenuate oxidative stress [28]. Hence, oxidative stress plays an indispensable role in impairing cardiac contractility through oxidative modifications of ion channels, pumps, or other transporter types, which alter their activities and eventually lead to impaired ECC.

\section{Oxidative stress and mitochondria- dependent cardiomyocyte apoptosis}

Oxidative stress is assumed to be one of the main risk factors for triggering cardiomyocyte death through apoptosis or necrosis and is involved in the pathogenesis of apoptosis through regulating downstream signaling pathways [29].
The changes of mitochondrial permeable transduction pore (mPTP) and membrane potential are an important early signal of oxidative stress response. The occurrence of oxidative stress and the consumption of ATP can induce mPTP long opening in the mitochondrial intima, and the decrease of proton gradient and potential energy, resulting in severe swelling of the mitochondria and the release of large amounts of cytochrome $\mathrm{c}$ and apoptosis-inducing factor, thus stimulating both caspase-dependent and caspaseindependent cascade apoptosis reaction, eventually promoting the occurrence and development of cardiovascular diseases. Maintaining the integrity of mitochondria and preventing the expression of apoptotic genetic programs are a feasible treatment for cardiomyocyte apoptosis. Oxidative stress-induced mitochondria-dependent apoptosis in cardiomyocyte is thought to involve three mechanisms: the activation of proapoptotic signaling pathways; suppression of antiapoptotic signaling pathways; and exerting an immediate ROS-induced effect on mitochondria that leads to the release of cytochrome $\mathrm{c}$.

\section{Mitochondria-related proapoptotic signaling}

Oxidative stress has a role in the pathogenesis of apoptosis as it can rapidly activate proapoptotic signaling pathways. Fluctuations in ROS generation have been demonstrated to activate a set of apoptotic signaling regulatory proteins, thereby mediating cellular responses [30]. Numerous studies have demonstrated that mitogen-activated protein kinases (MAPKs), c-Jun N-terminal kinases (JNKs), and p38 MAPK, regulator signals for cellular proliferation, differentiation, and survival, can be activated by stress and play crucial roles in mitochondria signal transduction during oxidative stress-induced apoptosis. There is also sufficient evidence to consider the possibility of targeting these signals-mitochondrial interactions in the prevention and treatment of heart disease. In the JNK and p38 pathways, initially, signal-regulating kinase 1 (ASK1), which is activated by oxidative stress, acts as a mitogenactivated protein kinase kinase kinase (MAPKKK) to phosphorylate and directly activate respective mitogenactivated protein kinase kinases (MAPKKs) of JNK and P38; these subsequently phosphorylate and activate JNK and $\mathrm{p} 38$, which then directly or indirectly regulate downstream apoptosis-related targets [31]. The p38/JNK and Janus kinase 2 (JAK2)/signal transducer and activator of transcription 3 (STAT3) pathways can not only be activated by ROS but also increase ROS generation, which is related to the elevated phosphorylation levels of p38/JNK and JAK2 [32]. Inhibition of mitochondrial JNK signal can attenuate the effect of ROS and mitochondrial dysfunction, thereby reducing the scope of myocardial infarction 
after ischemia-reperfusion [33]. ROS and JNK-dependent activation of mitochondrial proapoptotic pathway dominate the cardiomyocyte apoptosis induced by $\beta$-adrenergic receptor stimulation [34]. Moreover, spermine, an antioxidant, can effectively inhibit excessive oxidative stress and reduce cardiomyocyte apoptosis under high-glucose conditions through significantly suppressing the activation of the $\mathrm{p} 38 / \mathrm{JNK}$ and JAK2 pathways and results in decreased ROS generation, which can, conversely, suppress the phosphorylation of p38/JNK and JAK2 and eventually reduce ROS accumulation [32]. In addition, ASK1 in mitochondria can also act independently of JNK and P38 activation to induce apoptosis after being activated by oxidative stress [35]. Consequently, the JNK, p38, and ASK1 pathways all act as inducers of mitochondria-dependent apoptosis when activated by oxidative stress.

\section{Mitochondria related antiapoptotic signaling}

The imbalance between proapoptosis proteins and antiapoptosis protein has been demonstrated as the dominant cause for myocardial apoptosis related to oxidative stress [36]. Regarding the mitochondrial antiapoptotic signaling pathways, firstly, PI3K/AKT pathways, which is crucial for glucose uptake and insulin sensitivity, has been reported with markedly deactivation in high-fructose diet-induced metabolic syndrome [37]. The PI3K/AKT pathway has also been demonstrated as a vital antiapoptotic signaling pathway for cardiomyocytes in heart failure to attenuate myocardial apoptosis. To be more specific, the PI3K-AKT pathway of cardiomyocytes is activated by hypoxia; then, pAKT is transferred from the cytoplasm to the mitochondria, resulting in a significant increase of cytochrome c oxidase activity, thereby inhibiting cell apoptosis [38]. In a study of the effect of high fructose on cardiac apoptosis and survival pathways, it was found that compared with the control group, rats in the fructose-induced metabolic syndrome group (FIMS) had more apoptotic cells, and activated cytoplasmic cytochrome c, caspase- 3 and 9 levels (mitochondrial pathway) significantly increased; p-PI3K, p-Akt, and Bcl-2 protein levels significantly decreased. Activated cardiac mitochondrial-dependent apoptosis pathways have taken an important part of the responsibility in the pathogenesis of high-fructose diet-induced heart failure [39]. The activation of PI3K/AKT signaling pathway also results in phosphorylation of the downstream signaling like glycogen synthase kinase-3 $\beta$ (GSK3 $\beta$ ) and Nrf2 [36]. Subsequently, the PI3K/AKT/GSK3 $\beta$ pathway has been pointed out the crucial roles in acute myocardial infarction to inhibit cardiomyocyte apoptosis by increasing expression of antiapoptosis protein, B-cell lymphoma-2 (Bcl-2), and reducing the expressions of proapoptosis proteins including Bax and cleaved-caspase 3 [40]. A recent study, which was conducted with Sprague Dawley rats suffering heart failure after acute myocardial infarction, indicated that Qiliqiangxin capsule, a traditional Chinese medicine with cardioprotective effects, seems to maintain cardiac function and protect cardiomyocytes from mitochondria-dependent apoptosis induced by oxidative stress through activating the PI3K/AKT signaling and upregulating phospho-GSK3 $\beta$ [36]. Secondly, the MAPK/extracellular signal-regulated kinases (ERK1 and ERK2) signaling pathway is also involved in cardiac protection by preventing myocardial cell apoptosis in myocardial ischemia/reperfusion injury via the activation of pathway [41]. For example, DJ-1, as a multifunctional protein with antioxidant properties, exerts cytoprotective effects under conditions of oxidative stress by mediating cell survival and proliferation through activating the ERK1/2 pathway, as well as by suppressing apoptosis through the inhibiting of ASK1 activation [42]. Deng and Kang's studies showed that the activated ERK1/2 colocalize with mitochondria, and then phosphorylate Bcl-2 and Bad, thereby participating in the antiapoptotic effect mediated by mitochondria [43, 44]. Thus, it is likely that oxidative stress suppresses these mitochondria-regulated antiapoptotic signaling pathways and leads to the imbalance between proapoptotic signaling and antiapoptotic signaling, resulting in cardiomyocyte apoptosis. However, the existing researches exploring mitochondria antiapoptotic signaling principally focus on the model of myocardial infarction. The models such as MetS-related CVD and diabetic cardiomyopathy need to be adopted more widely to observe the alteration and roles of antiapoptotic signaling in further research.

\section{The release of cytochrome c}

Cytochrome c, an evolutionarily conserved and nuclearencoded mitochondrial protein, is essential not only for energy production but also for apoptosome formation and apoptotic cascade [45]. Regarding the specific mitochondrial-dependent apoptosis process, cytochrome c firstly separates from cardiolipin, the membrane-anchoring lipid to which cytochrome $\mathrm{c}$ is tethered, and then released into the extramitochondrial environment via mitochondrial permeability pores following the permeabilization of the outer mitochondrial membrane. Once released into the cytosol, cytochrome c complexes with dATP and apoptotic protease-activating factor 1 (APAF-1), resulting in an increase in the affinity of the complex for dATP, oligomerization of APAF-1, and subsequent apoptosome formation. As soon as the complex is formed, caspase-9 is activated, which initiates the hydrolytic cascade and leads to the activation of the effector caspases, and, eventually, to apoptosis [46]. Excessive ROS generation in cardiomyocytes results in excessive cytochrome $\mathrm{c}$ release which is the irreversible point in the cascade of events leading to apoptosis. The release of cytochrome $\mathrm{c}$ in cardiomyocytes occurs as a 
result of multiple events comprising ROS production, cardiolipin peroxidation, and $\mathrm{Ca}^{2+}$ overload in mitochondria [45]. Recently, it has been suggested that compounds such as melatonin can prevent cardiolipin peroxidation and protect mitochondria from excessive exposure to $\mathrm{Ca}^{2+}$ overload and limit reperfusion injury in the heart [47]. Another recent study has indicated the underlying mechanism of mesalazine resulting in cardiotoxic effect seems to relate to cytochrome c release, which means that mesalazine induces ROS formation leading to mitochondrial swell, mitochondrial membrane permeabilize, cytochrome c release, and eventual cardiomyocyte apoptosis [48]. However, as this study was conducted in isolated mitochondria, further studies in cells, animals, and clinical trials are still required. Overexpressed proapoptosis proteins, like Bax, cytochrome c, APAF-1, caspase 9, caspase 3 , and more, play vital roles in this mitochondrion-dependent apoptosis pathway induced by oxidative stress. Specifically, excessive Bax in cytoplasm could enter mitochondria and result in disrupting mitochondrial membrane permeability, which eventually makes excessive cytochrome $\mathrm{c}$ release from mitochondria into cytoplasm [49]. On the other hand, a study conducted in isoproterenol-induced myocardial-infarcted rats recently revealed that $\alpha$-bisabolol can abrogate the intrinsic pathway of apoptosis via reducing the expressions of these myocardial proapoptotic factors mentioned above and raising expression of antiapoptotic protein Bcl-2 [50]. Similarly, the cardiac protective effect of coccomyxagloeobotrydiformis has also been demonstrated in Mets rats with cardiovascular complications to base on facilitating the expression of Bcl-2 while suppressing the expression of Bax and cysteine protease caspase-3, protecting cardiomyocytes against apoptosis under oxidative stress [51]. Although the cardiac protective effects of $\alpha$-bisabolol and coccomyxagloeobotrydiformis have been demonstrated in rats, there should be further studies to translate this therapeutic effect in humans as well as demonstrate the efficacy and safety profile in clinical treatment.

\section{Conclusion}

Abundant experimental evidence indicates that myocardial oxidative stress generated under a variety of cardiac conditions plays a vital role in the etiopathogenesis of cardiac dysfunction in MetS. The mechanisms summarized in this review include excessive ROS-mediated mitochondrial abnormality, cardiomyocyte contractile/diastolic dysfunction, and mitochondria dependent apoptosis. Although the last few decades have seen substantial research efforts devoted to developing better therapeutic drugs to treat heart failure, the effects of currently available therapies on heart failure outcomes including mortality remain unsatisfactory. Hence, identifying the optimum intracellular nodes which aims to slow, inhibit, or even reverse the progress of cardiac dysfunction and degeneration has been one of the main challenges for optimizing therapies. Understanding the fundamental mitochondria mechanisms underlying cardiac dysfunction in MetS could not only lead to great advances in both preventative and therapeutic treatment of cardiovascular diseases but also promote the advancement of antioxidant therapies.

Acknowledgement We would like to thank all the doctors of China Medical University-The Queen's University of Belfast Joint College for their support and advice throughout this manuscript.

Authors contributions Aoxue Li and Ningning Zheng had the idea for the article, performed the literature search and data analysis, and drafted. All authors commented and critically revised the previous versions of the manuscript. All authors read and approved the final manuscript.

Funding The work was supported by the National Natural Science Foundation of China (FUND\#81800763) and the Natural Science Foundation of Liaoning Province of China (FUND\#20180551076).

Data availability All authors confirmed that all data and materials as well as software application support their published claims and comply with field standards.

\section{Declarations}

Ethics approval The manuscript does not contain clinical studies or patient data.

Conflict of interest All authors declare no competing interests.

Open Access This article is licensed under a Creative Commons Attribution 4.0 International License, which permits use, sharing, adaptation, distribution and reproduction in any medium or format, as long as you give appropriate credit to the original author(s) and the source, provide a link to the Creative Commons licence, and indicate if changes were made. The images or other third party material in this article are included in the article's Creative Commons licence, unless indicated otherwise in a credit line to the material. If material is not included in the article's Creative Commons licence and your intended use is not permitted by statutory regulation or exceeds the permitted use, you will need to obtain permission directly from the copyright holder. To view a copy of this licence, visit http://creativecommons.org/licenses/by/4.0/.

\section{References}

1. Ilkun O, Boudina S (2013) Cardiac dysfunction and oxidative stress in the metabolic syndrome: an update on antioxidant therapies. Curr Pharm Des 19:4806-4817. https://doi.org/10.2174/ 1381612811319270003

2. Bugger H, Abel ED (2008) Molecular mechanisms for myocardial mitochondrial dysfunction in the metabolic syndrome. Clin Sci (Lond) 114:195-210. https://doi.org/10.1042/CS20070166

3. Goffart S, von Kleist-Retzow JC, Wiesner RJ (2004) Regulation of mitochondrial proliferation in the heart: power-plant failure contributes to cardiac failure in hypertrophy. Cardiovasc Res 64:198-207. https://doi.org/10.1016/j.cardiores.2004.06.030 
4. Ventura-Clapier R, Garnier A, Veksler V (2004) Energy metabolism in heart failure. J Physiol 555:1-13. https://doi.org/10.1113/ jphysiol.2003.055095

5. Wei YH, Lu CY, Wei CY et al (2001) Oxidative stress in human aging and mitochondrial disease-consequences of defective mitochondrial respiration and impaired antioxidant enzyme system. Chin J Physiol 44:1-11

6. Williams RS (2000) Canaries in the coal mine: mitochondrial DNA and vascular injury from reactive oxygen species. Circ Res 86:915-916

7. Ide T, Tsutsui H, Hayashidani S et al (2001) Mitochondrial DNA damage and dysfunction associated with oxidative stress in failing hearts after myocardial infarction. Circ Res 88:529-535. https:// doi.org/10.1161/01.res.88.5.529

8. Clayton DA (1984) Transcription of the mammalian mitochondrial genome. Annu Rev Biochem 53:573-594. https://doi.org/10.1146/ annurev.bi.53.070184.003041

9. Wallace DC (1999) Mitochondrial diseases in man and mouse. Science 283:1482-1488. https://doi.org/10.1126/science.283.5407.1482

10. Ballinger SW, Patterson C, Yan CN et al (2000) Hydrogen peroxide- and peroxynitrite-induced mitochondrial DNA damage and dysfunction in vascular endothelial and smooth muscle cells. Circ Res 86:960-966. https://doi.org/10.1161/01.res.86.9.960

11. Scheffler K, Bjørås KØ, Bjørås M (2019) Diverse functions of DNA glycosylases processing oxidative base lesions in brain. DNA Repair (Amst) 81:102665. https://doi.org/10.1016/j.dnarep. 2019.102665

12. Islam MT (2017) Oxidative stress and mitochondrial dysfunctionlinked neurodegenerative disorders. Neurol Res 39:73-82. https:// doi.org/10.1080/01616412.2016.1251711

13. Zhang J, Wang X, Vikash V et al (2016) ROS and ROS-mediated cellular signaling. Oxid Med Cell Longev 2016:4350965. https:// doi.org/10.1155/2016/4350965

14. Kowalska M, Piekut T, Prendecki M et al (2020) Mitochondrial and nuclear DNA oxidative damage in physiological and pathological aging. DNA Cell Biol 39:1410-1420. https://doi.org/10. 1089/dna.2019.5347

15. Shokolenko I, Venediktova N, Bochkareva A et al (2009) Oxidative stress induces degradation of mitochondrial DNA. Nucleic Acids Res 37:2539-2548. https://doi.org/10.1093/nar/gkp100

16. Hamblin M, Friedman DB, Hill S et al (2007) Alterations in the diabetic myocardial proteome coupled with increased myocardial oxidative stress underlies diabetic cardiomyopathy. J Mol Cell Cardiol 42:884-895. https://doi.org/10.1016/j.yjmcc.2006.12.018

17. Dai DF, Hsieh EJ, Liu Y et al (2012) Mitochondrial proteome remodelling in pressure overload-induced heart failure: the role of mitochondrial oxidative stress. Cardiovasc Res 93:79-88. https:// doi.org/10.1093/cvr/cvr274

18. Bugger H, Schwarzer M, Chen D et al (2010) Proteomic remodelling of mitochondrial oxidative pathways in pressure overloadinduced heart failure. Cardiovasc Res 85:376-384. https://doi.org/ 10.1093/cvr/cvp344

19. Balderas-Villalobos J, Molina-Muñoz T, Mailloux-Salinas P et al (2013) Oxidative stress in cardiomyocytes contributes to decreased SERCA2a activity in rats with metabolic syndrome. Am J Physiol Heart Circ Physiol 305:H1344-H1353. https://doi. org/10.1152/ajpheart.00211.2013

20. Sag CM, Wagner S, Maier LS (2013) Role of oxidants on calcium and sodium movement in healthy and diseased cardiac myocytes. Free Radic Biol Med 63:338-349. https://doi.org/10. 1016/j.freeradbiomed.2013.05.035

21. Kiyuna LA, Albuquerque RPE, Chen $\mathrm{CH}$ et al (2018) Targeting mitochondrial dysfunction and oxidative stress in heart failure: challenges and opportunities. Free Radic Biol Med 129:155-168. https://doi.org/10.1016/j.freeradbiomed.2018.09.019
22. Fan M, Zhang J, Tsai CW et al (2020) Structure and mechanism of the mitochondrial $\mathrm{Ca}(2+)$ uniporter holocomplex. Nature 582:129-133. https://doi.org/10.1038/s41586-020-2309-6

23. Zima AV, Blatter LA (2006) Redox regulation of cardiac calcium channels and transporters. Cardiovasc Res 71:310-321. https://doi.org/10.1016/j.cardiores.2006.02.019

24. Sharikabad MN, Ostbye KM, Lyberg T, Brørs O (2001) Effect of extracellular $\mathrm{Mg}(2+)$ on $\mathrm{ROS}$ and $\mathrm{Ca}(2+)$ accumulation during reoxygenation of rat cardiomyocytes. Am J Physiol Heart Circ Physiol 280:H344-H353. https://doi.org/10.1152/ajpheart.2001.280.1.H344

25. Cooper LL, Li W, Lu Y et al (2013) Redox modification of ryanodine receptors by mitochondria-derived reactive oxygen species contributes to aberrant $\mathrm{Ca} 2+$ handling in ageing rabbit hearts. $\mathrm{J}$ Physiol 591:5895-5911. https://doi.org/10.1113/jphysiol.2013. 260521

26. Beutner G, Sharma VK, Giovannucci DR et al (2001) Identification of a ryanodine receptor in rat heart mitochondria. J Biol Chem 276:21482-21488. https://doi.org/10.1074/jbc.M101486200

27. Rowe GT, Manson NH, Caplan M, Hess ML (1983) Hydrogen peroxide and hydroxyl radical mediation of activated leukocyte depression of cardiac sarcoplasmic reticulum. Participation of the cyclooxygenase pathway. Circ Res 53:584-591. https://doi. org/10.1161/01.res.53.5.584

28. Monsalvo-Villegas A, Osornio-Garduño DS, Avila G (2018) Long-term regulation of excitation-contraction coupling and oxidative stress in cardiac myocytes by pirfenidone. Front Physiol 9:1801. https://doi.org/10.3389/fphys.2018.01801

29. Kumar D, Lou H, Singal PK (2002) Oxidative stress and apoptosis in heart dysfunction. Herz 27:662-668. https://doi.org/10. 1007/s00059-002-2430-3

30. Wang X, Martindale JL, Liu Y, Holbrook NJ (1998) The cellular response to oxidative stress: influences of mitogen-activated protein kinase signalling pathways on cell survival. Biochem $\mathbf{J}$ 333 ( Pt 2:291-300. https://doi.org/10.1042/bj3330291

31. Papaconstantinou $\mathbf{J}$ (2019) The role of signaling pathways of inflammation and oxidative stress in development of senescence and aging phenotypes in cardiovascular disease. Cells 8. https:// doi.org/10.3390/cells8111383

32. He Y, Yang J, Li H et al (2015) Exogenous spermine ameliorates high glucose-induced cardiomyocytic apoptosis via decreasing reactive oxygen species accumulation through inhibiting p38/ JNK and JAK2 pathways. Int J Clin Exp Pathol 8:15537-15549

33. Chambers JW, Pachori A, Howard S et al (2013) Inhibition of JNK mitochondrial localization and signaling is protective against ischemia/reperfusion injury in rats. J Biol Chem 288:4000-4011. https://doi.org/10.1074/jbc.M112.406777

34. Remondino A, Kwon SH, Communal C et al (2003) Beta-adrenergic receptor-stimulated apoptosis in cardiac myocytes is mediated by reactive oxygen species/c-Jun NH2-terminal kinase-dependent activation of the mitochondrial pathway. Circ Res 92:136-138. https:// doi.org/10.1161/01.res.0000054624.03539.b4

35. Liu T, Zhou HJ, Min W (2017) ASK family in cardiovascular biology and medicine. Adv Biol Regul 66:54-62. https://doi.org/ 10.1016/j.jbior.2017.10.011

36. Zhao Q, Li H, Chang L et al (2019) Qiliqiangxin attenuates oxidative stress-induced mitochondrion-dependent apoptosis in cardiomyocytes via PI3K/AKT/GSK3 $\beta$ signaling pathway. Biol Pharm Bull 42:1310-1321. https://doi.org/10.1248/bpb.b19-00050

37. Ajala-Lawal RA, Aliyu NO, Ajiboye TO (2020) Betulinic acid improves insulin sensitivity, hyperglycemia, inflammation and oxidative stress in metabolic syndrome rats via PI3K/Akt pathways. Arch Physiol Biochem 126:107-115. https://doi.org/10. 1080/13813455.2018.1498901

38. Song HP, Chu ZG, Zhang DX et al (2018) PI3K-AKT pathway protects cardiomyocytes against hypoxia-induced apoptosis by MitoKATP-mediated mitochondrial translocation of pAKT. Cell 
Physiol Biochem Int J Exp Cell Physiol Biochem Pharmacol 49:717-727. https://doi.org/10.1159/000493037

39. Cheng SM, Cheng YJ, Wu LY et al (2014) Activated apoptotic and anti-survival effects on rat hearts with fructose induced metabolic syndrome. Cell Biochem Funct 32:133-141. https://doi.org/10. $1002 /$ cbf.2982

40. Xu L, Jiang X, Wei F, Zhu H (2018) Leonurine protects cardiac function following acute myocardial infarction through antiapoptosis by the PI3K/AKT/GSK3 $\beta$ signaling pathway. Mol Med Rep 18:1582-1590. https://doi.org/10.3892/mmr.2018.9084

41. Chen Y, Ba L, Huang W et al (2017) Role of carvacrol in cardioprotection against myocardial ischemia/reperfusion injury in rats through activation of MAPK/ERK and Akt/eNOS signaling pathways. Eur J Pharmacol 796:90-100. https://doi.org/10.1016/j. ejphar.2016.11.053

42. Oh SE, Mouradian MM (2018) Cytoprotective mechanisms of DJ-1 against oxidative stress through modulating ERK1/2 and ASK1 signal transduction. Redox Biol 14:211-217. https://doi. org/10.1016/j.redox.2017.09.008

43. Deng X, Ruvolo P, Carr B, May WSJ (2000) Survival function of ERK1/2 as IL-3-activated, staurosporine-resistant Bcl2 kinases. Proc Natl Acad Sci U S A 97:1578-1583. https://doi.org/10.1073/ pnas.97.4.1578

44. Kang BPS, Urbonas A, Baddoo A et al (2003) IGF-1 inhibits the mitochondrial apoptosis program in mesangial cells exposed to high glucose. Am J Physiol Renal Physiol 285:F1013-F1024. https://doi.org/10.1152/ajprenal.00209.2003

45. Hüttemann M, Pecina P, Rainbolt M et al (2011) The multiple functions of cytochrome $\mathrm{c}$ and their regulation in life and death decisions of the mammalian cell: From respiration to apoptosis. Mitochondrion 11:369-381. https://doi.org/10.1016/j.mito.2011.01.010
46. Teringova E, Tousek P (2017) Apoptosis in ischemic heart disease. J Transl Med 15:87. https://doi.org/10.1186/s12967-017-1191-y

47. Petrosillo G, Moro N, Ruggiero FM, Paradies G (2009) Melatonin inhibits cardiolipin peroxidation in mitochondria and prevents the mitochondrial permeability transition and cytochrome c release. Free Radic Biol Med 47:969-974. https://doi.org/10. 1016/j.freeradbiomed.2009.06.032

48. Salimi A, Bahreini F, Jamali Z, Pourahmad J (2020) Mesalazine induces oxidative stress and cytochrome $\mathrm{c}$ release in isolated rat heart mitochondria: an analysis of cardiotoxic effects. Int J Toxicol 39:241-247. https://doi.org/10.1177/1091581820918163

49. Kumar P, Bhadauria AS, Singh AK, Saha S (2018) Betulinic acid as apoptosis activator: Molecular mechanisms, mathematical modeling and chemical modifications. Life Sci 209:24-33. https://doi.org/10.1016/j.lfs.2018.07.056

50. Nagoor Meeran MF, Laham F, Azimullah S et al (2019) $\alpha$-Bisabolol abrogates isoproterenol-induced myocardial infarction by inhibiting mitochondrial dysfunction and intrinsic pathway of apoptosis in rats. Mol Cell Biochem 453:89-102. https://doi. org/10.1007/s11010-018-3434-5

51. Zheng N, Ding X, Wei D et al (2018) Therapeutic effects of coccomyxagloeobotrydiformis on the metabolic syndrome in rats. Cell Physiol Biochem 48:1519-1529. https://doi.org/10.1159/000492262

Publisher's Note Springer Nature remains neutral with regard to jurisdictional claims in published maps and institutional affiliations. 